\title{
Non-ideality in Born-free energy of solvation in alcohol-water and dimethylsulfoxide-acetonitrile mixtures: Solvent size ratio and ion size dependence
}

\author{
HEMANT K KASHYAP and RANJIT BISWAS* \\ Department of Chemical, Biological and Macromolecular Sciences, and Unit for Nanoscience and \\ Technology, S.N. Bose National Centre for Basic Sciences, JD Block, Sector III, Salt Lake City, \\ Kolkata 700098 \\ e-mail: ranjit@bose.res.in
}

MS received 7 June 2007; accepted 12 July 2007

\begin{abstract}
Recent extension of mean spherical approximation (MSA) for electrolyte solution has been employed to investigate the non-ideality in Born-free energy of solvation of a rigid, mono-positive ion in binary dipolar mixtures of associating (ethanol-water) and non-associating (dimethylsulfoxideacetonitrile) solvents. In addition to the dipole moments, the solvent size ratio and ion size have been treated in a consistent manner in this extended MSA theory for the first time. The solvent-solvent size ratio is found to play an important role in determining the non-ideality in these binary mixtures. Smaller ions such as $\mathrm{Li}^{+}$and $\mathrm{Na}^{+}$show stronger non-ideality in such mixtures compared to bigger ions (for example, $\mathrm{Cs}^{+}$and $\mathrm{Bu}_{4} \mathrm{~N}^{+}$). The partial solvent polarization densities around smaller ions in tertiary butanol (TBA)water mixture is found to be very different from that in other alcohol-water mixtures as well as to that for larger ions in aqueous solutions of TBA. Non-ideality is weaker in mixtures consisting of solvent species possessing nearly equal diameters and dipole moments and is reflected in the mole fraction dependent partial solvent polarization densities.
\end{abstract}

Keywords. Non-ideality; Born-energy; dipolar-mixtures; unequal size; MSA.

\section{Introduction}

Solvent mixtures are important reaction media as one can tune the polarity, solubility, etc. of a solution by altering the composition. In the traditional barrier crossing model where an activation barrier separates the reactant and product, the reaction time scale is given by the rate at which the reactant crosses the barrier frequency. ${ }^{1-22}$ Solvation environment around a reactant affects the barrier-crossing rate both via solvent arrangement and time-dependent re-arrangement of solvent particles. ${ }^{1-22}$ As inter-diffusion and preferential solvation significantly slows down the dynamics in binary mixtures than in pure solvents, ${ }^{23-40}$ solvent effects on chemical reactions are expected to be different in binary liquid mixtures. In addition to simple chemical reactions, transport property such as ion mobility is also significantly modified in binary mixtures. For example, limiting ionic conductivity of uni-positive alkali metal ions tertiary butanol (TBA)-

\footnotetext{
*For correspondence
}

water exhibits a non-monotonic alcohol mole fraction dependence. ${ }^{41}$ Moreover, bigger ion such as tetrabutyl ammonium ion $\left(\mathrm{Bu}_{4} \mathrm{~N}^{+}\right)$shows a linear mole fraction dependence in the same mixture. ${ }^{41}$ This different composition dependence originates partly from the nature of solvation of these individual ions and partly from the modification of the solution dynamics. In order to understand solvent effects on these simple events in binary mixtures, one therefore needs to investigate the solution structure in terms of intraand inter-molecular liquid correlations and modifications of solution dynamics.

Anomalous physical properties of alcohol-water mixtures at room temperature have been studied extensively over the years. ${ }^{42-69}$ Different structures and mechanism for formation of those structures in alcohol-water solutions at low to high alcohol concentrations have been proposed. ${ }^{42-69}$ Several experimental studies indicate that at low alcohol content water structure is stronger than that in pure water. $^{70-72}$ This is then used to explain the anomalous decrease in entropy, ${ }^{42,43}$ anomalous increase in sound 
attenuation coefficient ${ }^{44}$ and emergence of an endothermic peak in the partial heat of solution of several crystalline salts in aqueous alcohol solutions..$^{70,71}$ Further experimental support ${ }^{72}$ to this idea came from the fact that partial molal heat of solution showed an even larger endothermic peak when the relevant experiments were carried out at $\sim 277 \mathrm{~K}$. At higher alcohol concentration, however, the water structure breaks down and the characteristic chainlike structure of pure alcohol predominates. ${ }^{50-72} \mathrm{At}$ high water content, an infinitely dilute third component (an ion or a neutral solute) competes with the alcohol molecules to be properly 'surrounded' by water structure. ${ }^{70-72}$ This is dictated by the relative interaction strengths between the solute and the solvent molecules of different species. As a result, the structure forming or breaking ability of alcohol in water is modified in presence of an ion or a solute. ${ }^{70-72}$ Moreover, specific interactions among these components may favour enriching of one component over the other in the first few solvation shells giving rise to what is known as preferential solvation. In such a situation one would like to ask the following questions. First, will there be any preferential solvation and thus non-ideality in the absence of any solutesolvent and solvent-solvent specific interactions? This can indeed be a situation as evidenced by the recent simulation studies of non-ideality in viscosity for model binary mixtures. ${ }^{73,74}$ Second, how solvent size disparity would affect the preferential solvation and thus the non-ideality? Third, what are the effects of solute size on the non-ideality? Fourth, can one develop a theoretical formalism which will be simple and analytically tractable yet capable of describing the liquid structure in these complex mixtures, at least, qualitatively?

Recently, we have extended the mean spherical approximation $^{75}$ (MSA) framework in order to investigate answers to these questions. The extended MSA incorporates the difference in solvent diameters and dipole moments as well as ion size systematically for the first time. ${ }^{76}$ In this article, we apply this extended MSA formalism to study the solvent size ratio dependence of non-ideality in Born-free energy of solvation of an infinitely dilute ion in two different types of binary mixtures at different mole fraction of the solvent components: mixtures containing associating (that is, H-bonding) solvents and those made up of non-associating solvents. We consider mono-hydroxy alcohol such as ethanol and water in the first category, and dimethyl sulfoxide (DMSO) mix- ture and acetonitrile in the second. The DMSOacetonitrile is interesting because the larger component (DMSO) possesses higher dipole moment. ${ }^{5,77}$ This leads to a competition in packing around a dissolved ion in DMSO-acetonitrile mixture. Moreover, the molecular diameters of these solvents are similar $^{5,77}$ and hence the non-ideality in polarization structure (and hence in Born-free energy of solvation) is expected to be weaker. In contrast, the dipole moment of water is slightly larger than the alcohol studied here but the size is considerably smaller. This assists water molecules to be preferably chosen in the first solvation shell and hence the non-ideality is likely to be stronger. Therefore, larger ions would be able to accommodate the larger solvent components leading to a more 'homogeneous' solvation structure. This, in turn, will render the non-ideality weaker. It is to be noted here that the preferential solvation in model binary mixtures have been studied earlier. ${ }^{78,79}$ In these studies either the size disparity of all the species was not treated systematically or the solvent size ratio was not incorporated. However, these studies indicated that specific interactions among the components are not crucial for giving rise to non-ideality. ${ }^{78}$

In the present, work we are studying solvent structure around an ion in a binary mixture of fluids made up of dipolar hard spheres with unequal radii and dipole moments. ${ }^{80-87}$ We have, therefore, used the MSA frameworks provided by Isbister and Bearman, ${ }^{82}$ Chan et $a l^{80}$ and Blum et $a l^{84-87}$. Here we model the solvent molecules of different species as dipolar hard spheres with single point dipoles located at the centers. ${ }^{80}$ Ions are considered as hard spheres carrying point charges of one unit at the respective centers. ${ }^{80}$ The ions are present at infinite dilution. The electroneutrality is maintained, as usually done in the MSA. The molecular diameters of solvent molecules have been calculated from the van der Waals' space filling model. ${ }^{77}$ Experimental values of dipole moments are used to specify different solvent components. ${ }^{5}$ Experimental crystallographic ionic radii are used to denote the sizes of the ions studied. ${ }^{5}$ Mole fraction dependent densities are taken from relevant experiments ${ }^{44,88-89}$ and used to calculate the mole fraction dependent properties of these binary mixtures. As we use the MSA framework in our formalism, the analytical tractability of the former is retained. This makes the present theory simple to use in cases where intra and inter-molecular correlations are required as inputs. However, the correlations obtained 
by the present theory would be at best semi-quantitative as MSA is known to work rather poorly for strongly dipolar systems. ${ }^{80}$

The organization of the rest of the paper is as follows. We describe the theoretical formulation in the next section. This will be brief as the details have been given elsewhere. Numerical results and discussions are given in §III. Concluding remarks are provided in $§ I V$.

\section{Theoretical formulation}

We shall present here briefly the relevant theory as details have been discussed in ref. 76. Let us consider a solution of an electrolyte of equal-sized ions in a binary dipolar mixture. Let $R_{i}$ and $R_{j}$ be the hard sphere diameters of the two types of solvent molecules with dipole moments $\mu_{i}$ and $\mu_{j}$, respectively. The solvent number densities are $\rho_{i}$ and $\rho_{j}$. Here, $R_{i} \neq R_{j}$, and $\mu_{i} \neq \mu_{j}$. The electro-neutrality is maintained by setting

$$
\sum_{\alpha} \rho_{\alpha} z_{\alpha}=0
$$

where $\rho_{\alpha}$ denotes the number density of ionic species $\alpha$ with charge $z_{\alpha}$ and diameter $R_{\alpha}=R$. The interaction potentials are given by ${ }^{79-80}$

$$
\begin{gathered}
u_{\alpha \beta}(r)=z_{\alpha} z_{\beta} e^{2} / r, r>R_{\alpha \beta}=R, \\
u_{\alpha j}\left(\mathbf{r}, \omega_{2}\right)=-z_{\alpha} e \mu_{j} E_{2} / r^{2}, \\
r>R_{\alpha j}=\left(R+R_{j}\right) / 2 \\
u_{i \beta}\left(\omega_{1}, \mathbf{r}\right)=z_{\beta} e \mu_{i} E_{1} / r^{2}, \\
r>R_{i \beta}=\left(R_{i}+R\right) / 2 \\
u_{i j}\left(\omega_{1}, \mathbf{r}, \omega_{2}\right)=-\mu_{i} \mu_{j} D_{12} / r^{3}, \\
r>R_{i j}=\left(R_{i}+R_{j}\right) / 2,
\end{gathered}
$$

where $\alpha, \beta$ indices are for ions and $i, j$ indices are for dipoles. $D_{12}=\hat{\mu}\left(\omega_{1}\right) \cdot(\mathbf{3} \hat{\mathbf{r}} \hat{\mathbf{r}}-\mathbf{I}) \cdot \hat{\mu}\left(\omega_{2}\right)$, which arises due to the angle dependent parts of the interaction potentials. $E_{i}=\hat{\mu}\left(\omega_{i}\right) \cdot \hat{\mathbf{r}}$. In above equations, $e$ represents the elementary charge, I denotes a $3 \times 3$ unit tensor, and $\hat{\mathbf{r}}$ is the unit vector from the molecule denoted by the first index towards the one denoted by second index. The orientation of $i$ th type dipole is described by $\omega_{i}$.
Now the Ornstein-Zernike (OZ) relation can be used to obtain the MSA solution for the correlation functions in an electrolyte solution of binary dipolar mixture. ${ }^{80}$ The relevant $\mathrm{OZ}$ expressions along with the MSA closure relations are discussed in ref. 80 . Subsequently, the algebra described in Chan et al ${ }^{80}$ leads to the following expressions for the wave number $(k)$ and orientation dependent static correlation functions

$$
\begin{aligned}
& \tilde{h}_{\alpha \beta}(k)=\tilde{c}_{\alpha \beta}(k)+\sum_{\gamma} \rho_{\gamma} \tilde{c}_{\alpha \beta}(k) \tilde{h}_{\gamma \beta}(k)+, \\
& \sum_{k} \rho_{k}\left\langle\tilde{c}_{\alpha k}\left(\mathbf{k}, \omega_{3}\right) \tilde{h}_{k \beta}\left(\omega_{3}, \mathbf{k}\right)\right\rangle_{\omega_{3}} \\
& \tilde{h}_{\alpha j}\left(\mathbf{k}, \omega_{2}\right)=\tilde{c}_{\alpha j}\left(\mathbf{k}, \omega_{2}\right)+\sum_{\gamma} \rho_{\gamma} \tilde{c}_{\alpha \gamma}(k) \tilde{h}_{\gamma j}\left(\mathbf{k}, \omega_{2}\right)+ \\
& \sum_{k} \rho_{k}\left\langle\tilde{c}_{\alpha k}\left(\mathbf{k}, \omega_{3}\right) \tilde{h}_{k j}\left(\omega_{3}, \mathbf{k}, \omega_{2}\right)\right\rangle_{\omega_{3}} \\
& \tilde{h}_{i \beta}\left(\omega_{1}, \mathbf{k}\right)=\tilde{c}_{i \beta}\left(\omega_{1}, \mathbf{k}\right)+\sum_{\gamma} \rho_{\gamma} \tilde{c}_{i \gamma}\left(\omega_{1}, \mathbf{k}\right) \tilde{h}_{\gamma \beta}(k)+ \\
& \sum_{k} \rho_{k}\left\langle\tilde{c}_{i k}\left(\omega_{1}, \mathbf{k}, \omega_{3}\right) \tilde{h}_{k \beta}\left(\omega_{3}, \mathbf{k}\right)\right\rangle_{\omega_{3}}, \\
& \tilde{h}_{i j}\left(\omega_{1}, \mathbf{k}, \omega_{2}\right)=\tilde{c}_{i j}\left(\omega_{1}, \mathbf{k}, \omega_{2}\right)+ \\
& \sum_{\gamma} \rho_{\gamma} \tilde{c}_{i \gamma}\left(\omega_{1}, \mathbf{k}\right) \tilde{h}_{\gamma j}\left(\mathbf{k}, \omega_{2}\right)+ \\
& \sum_{k} \rho_{k}\left\langle\tilde{c}_{i k}\left(\omega_{1}, \mathbf{k}, \omega_{3}\right) \tilde{h}_{k j}\left(\omega_{3}, \mathbf{k}, \omega_{2}\right)\right\rangle_{\omega_{3} .}
\end{aligned}
$$

Carrying out the angular convolution in (3a-d) and using the charge neutrality condition, and setting

$$
\begin{aligned}
& \rho_{c}=\sum_{\gamma} \rho_{\gamma} z_{\gamma}^{2}, \\
& \rho_{I}=\sum_{\gamma} \rho_{\gamma},
\end{aligned}
$$

we obtain the following coupled set of equations for the correlation functions due to the electrostatic interactions between ions, ion-dipole and dipoles ${ }^{76}$

$$
\begin{aligned}
\tilde{h}_{\alpha \beta}^{C}(k)= & \tilde{c}_{\alpha \beta}^{C}(k)+\rho_{c} \tilde{c}_{\alpha \gamma}^{C}(k) \tilde{h}_{\gamma \beta}^{C}(k) \\
& -\frac{1}{3} \sum_{k} \rho_{k} \tilde{c}_{\alpha k}^{E}(k) \tilde{h}_{k \beta}^{E}(k)
\end{aligned}
$$




$$
\begin{aligned}
\tilde{h}_{\alpha j}^{E}(k)= & \tilde{c}_{\alpha j}^{E}(k)+\rho_{c} \tilde{c}_{\alpha \gamma}^{C}(k) \tilde{h}_{\gamma j}^{E}(k) \\
& +\frac{1}{3} \sum_{k} \rho_{k} \tilde{c}_{\alpha k}^{E}(k) \tilde{h}_{k j}^{+}(k) \\
\tilde{h}_{i \beta}^{E}(k)= & \tilde{c}_{i \beta}^{E}(k)+\rho_{c} \tilde{c}_{i \gamma}^{E}(k) \tilde{h}_{\gamma \beta}^{C}(k) \\
& +\frac{1}{3} \sum_{k} \rho_{k} \tilde{c}_{i k}^{+}(k) \tilde{h}_{k \beta}^{E}(k) \\
\tilde{h}_{i j}^{+}(k)= & \tilde{c}_{i j}^{+}(k)-\rho_{c} \tilde{c}_{i \gamma}^{E}(k) \tilde{h}_{\gamma j}^{E}(k) \\
& +\frac{1}{3} \sum_{k} \rho_{k} \tilde{c}_{i k}^{+}(k) \tilde{h}_{k j}^{+}(k) .
\end{aligned}
$$

Now using the algebra described in ref. 76 we obtain the following expression for the Born-free energy of solvation for an ion

$$
\begin{aligned}
E_{\mathrm{Born}} & =\sum_{k} \rho_{k} \int d \mathbf{r}\left\langle\Omega_{\alpha k}(\mathbf{r}, \omega) g_{\alpha k}(\mathbf{r}, \omega)\right\rangle_{\omega} \\
& =-\frac{4 \pi z_{\alpha}^{2} e}{3} \sum_{k} \rho_{k} \Omega_{k} \int_{R_{\alpha k}}^{\infty} d r h_{\alpha k}^{E}(r),
\end{aligned}
$$

where the second equality follows because $h_{\alpha k}^{E}(r)=0$ for $r<R_{\alpha k}$. We now need to find

$$
\int_{R_{\alpha k}}^{\infty} d r h_{\alpha k}^{E}(r)
$$

for evaluating $E_{\mathrm{Born}}$. This is calculated from the iondipole correlation function as follows ${ }^{79,80}$

$$
\begin{aligned}
H_{\alpha j}^{E}(x) & =2 \pi \int_{x}^{\infty} d r P_{1}(x / r) r h_{\alpha j}^{E}(r), \\
& =2 \pi x \int_{R_{\alpha j}}^{\infty} d r h_{\alpha j}^{E}(r)=x H 1_{\alpha j}^{E} \text { for } x \leq R_{\alpha j},
\end{aligned}
$$

where

$$
H 1_{\alpha j}^{E}=2 \pi \int_{R_{\alpha j}}^{\infty} d r h_{\alpha j}^{E}(r)
$$

is a constant that needs to be determined. Equation (7) and the above discussion now provide the fol- lowing expression of Born-energy $\left(E_{\text {Born }}\right)$ for an ion dissolved in binary mixture of dipolar solvents with unequal size and different dipole moments

$$
\begin{aligned}
E_{\text {Born }} & =-\frac{2 z_{\alpha}^{2} e}{3} \sum_{k} \rho_{k} \mu_{k} H 1_{\alpha k}^{E} \\
& =-\frac{2 z_{\alpha}^{2} e}{3} \sum_{k} \rho_{k} \mu_{k} H 1_{k \alpha}^{E} .
\end{aligned}
$$

Now we need to calculate $H 1_{k \alpha}^{E}\left(=H 1_{\alpha k}^{E}\right)$. Baxter factorization $^{80,90}$ gives for $x>S_{j \alpha}=\left(R_{j}-R_{\alpha}\right) / 2$

$$
\begin{aligned}
H_{j \alpha}^{E}(x)= & -Q_{j \alpha}^{E}(x) \\
& +\frac{1}{3} \sum_{k} \rho_{k} \int_{S_{j k}}^{R_{j k}} d y Q_{j k}^{+}(y) H_{k \alpha}^{E}(x-y) .
\end{aligned}
$$

From (8) when $S_{j \alpha}<x \leq R_{j \alpha}$

$$
\begin{aligned}
x H 1_{j \alpha}^{E}= & -Q_{j \alpha}^{E}(x) \\
& +\frac{1}{3} \sum_{k} \rho_{k} H 1_{k \alpha}^{E} \int_{S_{j k}}^{R_{j k}} d y Q_{j k}^{+}(y)(x-y),
\end{aligned}
$$

where $Q_{j k}^{+}(x)$ depends solely on the dipolar mixture properties and is given by, ${ }^{76,79,80}$

$$
Q_{i j}^{+}(x)=3\left[\frac{1}{2} a_{i j}\left(x-R_{i j}\right)\left(x-S_{i j}\right)+b_{i j}\left(x-R_{i j}\right)\right],
$$

where the values of $a_{i j}$ and $b_{i j}$ are described in ref. 76 .

Equation (11) consists of two coupled simultaneous equations in $H 1_{j \alpha}^{E}$ when $j=1,2$ and $\alpha$ is fixed (a given ionic species). Using Baxter's method ${ }^{90}$ we obtain the following expression for $C_{j \alpha}^{E}(x)$

$$
\begin{aligned}
C_{j \alpha}^{E}(x) & =-Q_{j \alpha}^{E}(x), x R_{j \alpha} \\
& +\frac{1}{3} \sum_{k} \rho_{k} \int_{S_{k j}}^{R_{k j}} d y Q_{k j}^{+}(y) Q_{k \alpha}^{E}(x+y), x R_{j \alpha}
\end{aligned}
$$

with closure relation

$$
C_{j \alpha}^{E}(x)=2 \pi e \beta \mu_{j}, \quad x \geq R_{j \alpha} .
$$

Therefore, from (13) and (14), we obtain 


$$
\begin{aligned}
2 \pi e \beta \mu_{j}= & -Q_{j \alpha}^{E}(x) \\
& +\frac{1}{3} \sum_{k} \rho_{k} \int_{S_{k j}}^{R_{k j}} d y Q_{k j}^{+}(y) Q_{k \alpha}^{E}(x+y), x \geq R_{j \alpha}
\end{aligned}
$$$$
=\frac{1}{3} \sum_{k} \rho_{k} \mu_{k}\left[\frac{1}{2 \pi r}\left\{\frac{1}{r} H_{k \alpha}^{E}(r)-\frac{d}{d r} H_{k \alpha}^{E}(r)\right\}\right],
$$

Equation (15) contains two coupled simultaneous equations in $Q_{j \alpha}^{E}(x)$ when $j=1,2$ for a fixed $\alpha$. Using the expressions of $Q_{j \alpha}^{E}(x)$ given in ref. 76 and carrying out the relevant algebra, from (11) we obtain the following expressions for as,

$$
\begin{gathered}
H 1_{1 \alpha}^{E}=\frac{-Q_{1 \alpha}^{E}\left(R_{1 \alpha}\right)\left[R_{2 \alpha}-R_{2 \alpha} I_{22}+\tilde{I}_{22}\right]-}{\left[R_{1 \alpha}-R_{1 \alpha} I_{11}+\tilde{I}_{11}\right]\left[R_{2 \alpha}-R_{2 \alpha} I_{22}+\tilde{I}_{22}\right]-}, \\
{\left[R_{1 \alpha} \bar{I}_{12}-\tilde{I}_{12}\right]\left[R_{1 \alpha} \bar{I}_{21}-\tilde{I}_{21}\right]} \\
H 1_{2 \alpha}^{E}=\frac{-Q_{2 \alpha}^{E}\left(R_{2 \alpha}\right)\left[R_{1 \alpha}-R_{1 \alpha} I_{11}+\tilde{I}_{11}\right]-}{\left[R_{1 \alpha}-R_{1 \alpha} I_{11}+\tilde{I}_{11}\right]\left[R_{2 \alpha}-R_{2 \alpha} I_{22}+\tilde{I}_{22}\right]-} \\
{\left[R_{1 \alpha} \bar{I}_{12}-\tilde{I}_{12}\right]\left[R_{1 \alpha} \bar{I}_{21}-\tilde{I}_{21}\right]}
\end{gathered}
$$

where $I_{i j}, \bar{I}_{i j}$ and $\tilde{I}_{i j}$ are given in ref. 76 .

Substitution of $H 1_{j \alpha}^{E}$ (from (16) and (17)) into (9) provides the required expression for $E_{\mathrm{Born}}$. Born free energy $\left(F_{\text {Born }}\right)$ of solvation is then calculated from the following relation ${ }^{80}$

$$
F_{\text {Born }}=\int_{0}^{e} d e\left(E_{\text {Born }} / 2\right) .
$$

The expressions for partial polarization densities of constituent solvents around an ion with charge $z_{\alpha} e$ at infinite dilution has been shown to be given by ${ }^{79,80}$

$$
\begin{aligned}
P_{\alpha}(r) & =\sum_{k} P_{\alpha k}(r) \\
& =\frac{1}{4 \pi} \sum_{k} \rho_{k} \Omega_{k} \int d \omega g_{\alpha k}(r, \omega)\left(\Omega_{\mathrm{k}}(\omega) \cdot \hat{\mathbf{r}}\right)
\end{aligned}
$$

where $P_{\alpha k}(r)$ is partial polarization density of $k$ th species. Since $h_{\alpha k}^{E}=h_{k \alpha}^{E}$ one can write the above equation as

$$
P_{\alpha}(r)=\frac{1}{3} \sum_{k} \rho_{k} \mu_{k} h_{\alpha k}^{E}(r)=\frac{1}{3} \sum_{k} \rho_{k} \mu_{k} h_{k \alpha}^{E}(r)
$$

where $H_{k \alpha}^{E}(r)$ is given by ${ }^{79,80}$

$$
\begin{gathered}
H_{j \alpha}^{E}(r)=r H 1_{j \alpha}^{E}, r \leq R_{j \alpha} \\
H_{j \alpha}^{E}(r)=-Q_{j \alpha}^{E}(r)+\frac{1}{3} \sum_{k} \rho_{k} \int_{S_{j k}}^{R_{j k}} d s Q_{j k}^{+}(s) H_{k \alpha}^{E}(r-s) \\
r>R_{j \alpha} . r>R_{j \alpha} .
\end{gathered}
$$

\section{Numerical results and discussion}

As discussed in the Introduction, we would now present numerical results of two different mixtures, namely; ethanol-water and DMSO-acetonitrile mixtures. The relevant parameters required for calculations are summarized in table 1 . All the calculations done here are at $298 \cdot 15 \mathrm{~K}$. The non-ideality in mole fraction $\left(x_{2}\right)$ dependent Born free energy of solvation of an ion is expressed as follows ${ }^{76,78,79}$

$$
\begin{aligned}
& \Delta F_{\text {Born }}\left(x_{2}\right)=F_{\text {Born }}\left(x_{2}\right)-F_{\text {Born }}\left(x_{2}=0\right) \\
& \quad-x_{2}\left[F_{\text {Born }}\left(x_{2}=1\right)-F_{\text {Born }}\left(x_{2}=0\right)\right] \\
& \quad=F_{\text {Born }}\left(x_{2}\right)-\left[\left(1-x_{2}\right) F_{\text {Born }}\left(x_{2}=0\right)+x_{2} F_{\text {Born }}\left(x_{2}=1\right)\right]
\end{aligned}
$$

The second equality of (22) indicates that the excess Born-free energy of solvation is the deviation in Born-free energy of solvation for an ion at a mole fraction $\left(x_{2}\right)$ in a binary mixture from the sum of the mole fraction weighted Born-free energies in the pure

Table 1. Solvent parameters used in the calculation.

\begin{tabular}{lcc}
\hline Solvent & $\begin{array}{c}\text { Dipole } \\
\text { moment (D) }\end{array}$ & $\begin{array}{c}\text { Diameter* } \\
\left(10^{-8} \mathrm{~cm}\right)\end{array}$ \\
\hline Water & 1.85 & $2 \cdot 80$ \\
Ethanol & 1.69 & $4 \cdot 78$ \\
Acetonitrile (ACN) & 3.5 & $4 \cdot 5$ \\
Dimethylsulfoxide (DMSO) & $4 \cdot 1$ & $5 \cdot 3$ \\
\hline
\end{tabular}

*The diameters of $\mathrm{Li}^{+}$and $\mathrm{C}_{4}$ ions used in the calculations are 1.24 and $10 \AA$, respectively 
components. We would use (22) to investigate the mole fraction dependent non-ideality in $\Delta F_{\text {Born }}$ in ethanol-water and DMSO-acetonitrile mixtures.

Figure 1 shows the mole fraction dependence of Born-free energy of solvation, $F_{\text {Born }}$ and excess Bornfree energy of solvation, $\Delta F_{\text {Born }}$ in these mixtures for two ions of very different diameters, $\mathrm{Li}^{+}$and tetrabutyl ammonium ion $\left(\mathrm{C}_{4}\right)$. The Born-free energy of solvation, $F_{\text {Born }}$ (upper panel) indicate different mole fraction dependence in ethanol-water and DMSO-acetonitrile mixtures. The insensitivity (or very weak dependence) of $F_{\text {Born }}$ on DMSO mole fraction in DMSOacetonitrile mixture (filled symbols) is a manifestation of competition between packing and electrostatic interactions. The dipole moment of DMSO is little larger than that of acetonitrile and hence iondipole interaction would favor DMSO more in number in the first solvation shell of an ion. However, packing constraint (repulsion) would not favour disfavor DMSO as its size is slightly larger than acetonitrile. Therefore, a delicate balance between these two interactions renders the mole fraction depend-
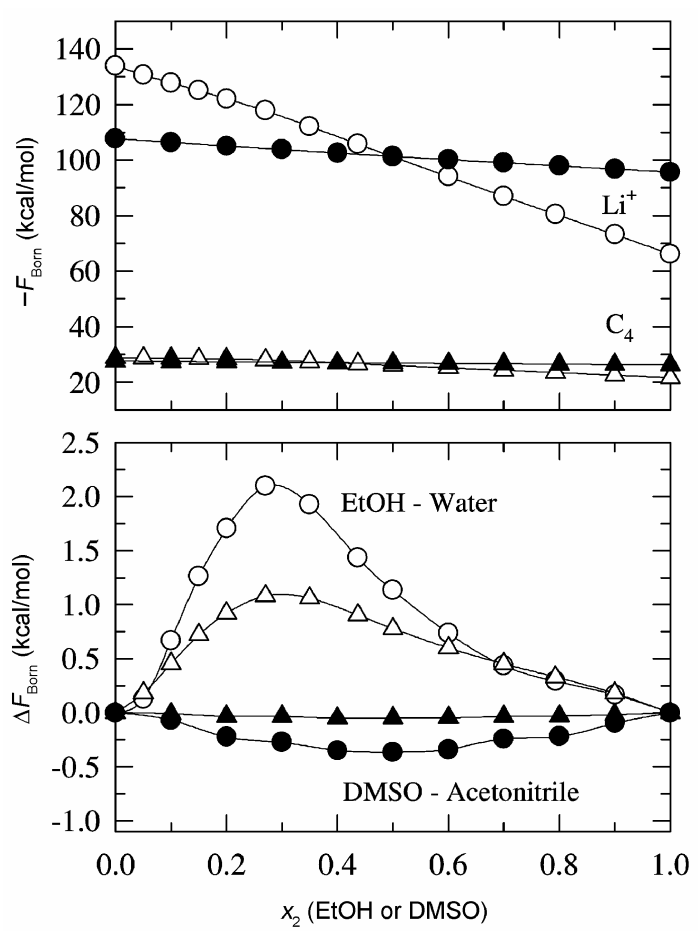

Figure 1. Born-free energy of solvation $F_{\text {Born }}$ (upper panel) and excess Born-free of solvation $\Delta F_{\text {Born }}$ (lower panel) for $\mathrm{Li}^{+}$(circles) and $\mathrm{C}_{4}$ (triangles) ions as a function of mole fraction of second (larger) component $\left(x_{2}\right)$ in ethanol-water (open symbols) and dimethylsulfoxideacetonitrile (solid symbols) mixtures. ence of $F_{\text {Born }}$ a very weak one. Note also that even in ethanol-water mixture (open symbols), the mole fraction dependence in $F_{\text {Born }}$ for $\mathrm{C}_{4}$ (triangles) is very weak. This indicates that $\mathrm{C}_{4}$ being large in size can accommodate larger solvent molecules more favourably than smaller ions, resulting much less concentration fluctuations in the nearest neighbour solvent arrangements. As expected, the values of $F_{\text {Born }}$ for these ions in pure solvents match exactly with predictions from the works of Chan et $a l^{80}$.

The lower panel of figure 1 shows the mole fraction dependence of the excess Born-free energy of solvation, $\Delta F_{\text {Born }}$ for $\mathrm{Li}^{+}$and $\mathrm{C}_{4}$ in ethanol-water and DMSO-acetonitrile mixtures. Note that the slope of $\Delta F_{\text {Born }}$ for DMSO-acetonitrile mixture is opposite to that found in ethanol-water mixtures. Similar results have also been found for smaller ions in TBA-water mixtures when we studied aqueous solutions of methanol and TBA. ${ }^{76}$ The positive values of $\Delta F_{\text {Born }}$ clearly indicates that DMSO molecules around a small ion such as $\mathrm{Li}^{+}$is increasingly disfavored due to
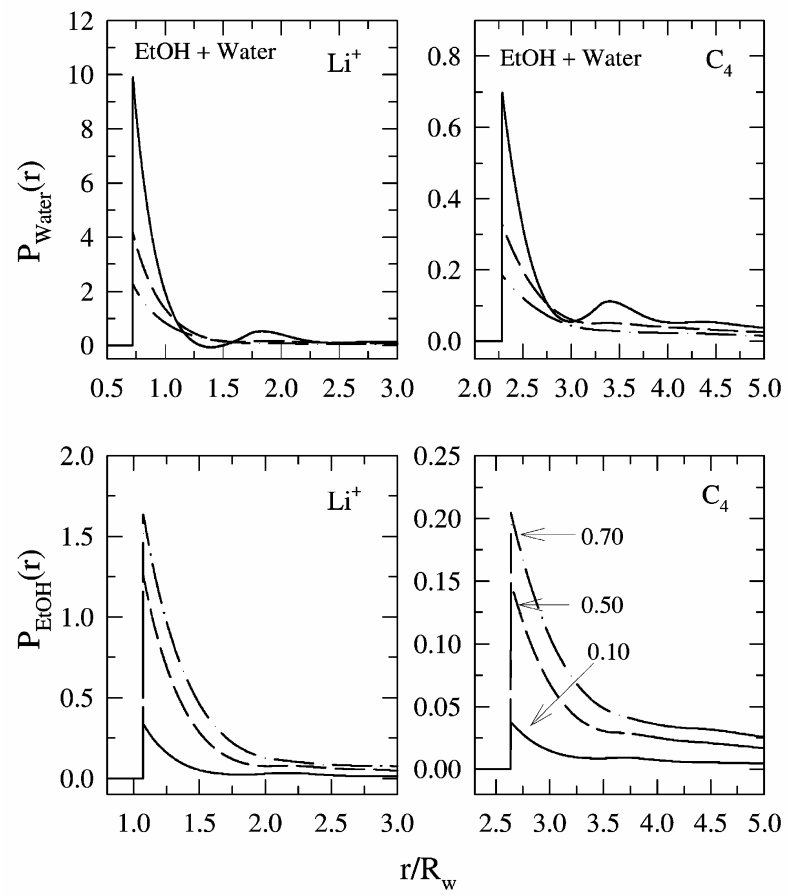

Figure 2. Partial polarization densities of water $P_{\text {water }}(r)$ and ethanol $P_{\mathrm{EtOH}}(r)$ (both scaled by $\sqrt{k_{B} T / R_{w}^{3}}$ ) around $\mathrm{Li}^{+}$and $\mathrm{C}_{4}$ ions as a function of distance $r$ (scaled by water diameter $R_{w}$ ) from the centre of the ion at $0 \cdot 10$ (solid line), 0.50 (short dashed line) and 0.70 (dotted dashed line) mole fractions of ethanol in ethanol-water mixtures. For discussion, see text. 
larger size. This is indeed a manifestation of dominance of repulsive interactions in determining the solvation structure around a solvated ion. Another important aspect of this figure (lower panel) is that the absolute magnitude of $\Delta F_{\text {Born }}$ is 4 times less for $\mathrm{Li}^{+}$in $\mathrm{DMSO}-$ acetonitrile mixture than that in ethanol-water solution. This indicates that the solvent size ratio indeed plays a crucial role in determining the extent of non-ideality in binary mixtures. This is one of the new results of the present scheme described here. Also, the position of the extremum of DMSO-acetonitrile curve occurs at almost 50:50 composition whereas that for ethanol-water occurs at $\sim 0.3 \mathrm{~mol}$ fraction of ethanol. This further proves that the solvent size ratio not only determines the extent of non-ideality in Born-free energy of solvation in binary mixtures, but determines also the location of the extremum on the mole fraction axis.

We next present the numerical results on the partial solvent polarization densities around $\mathrm{Li}^{+}$and $\mathrm{C}_{4}$ in these mixtures at three different mole fractions of either ethanol or DMSO. Figure 2 shows the partial solvent polarization densities in ethanol-water mix-
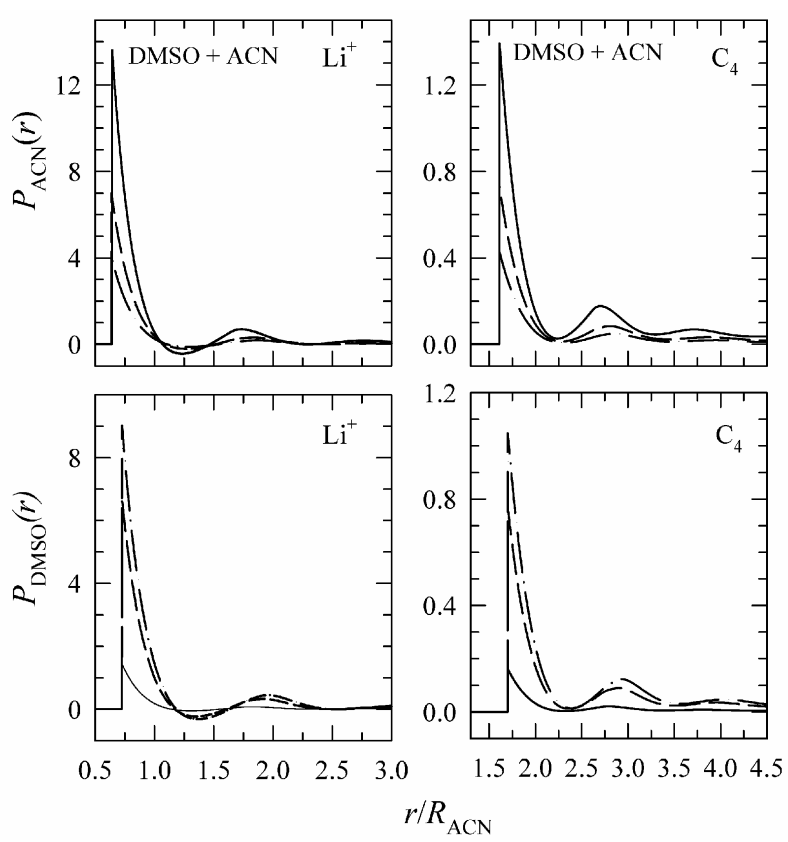

Figure 3. Partial polarization densities of acetonitrile $P_{\mathrm{ACN}}(r)$ and dimethylsulfoxide $P_{\mathrm{DMSO}}(r)$ (both scaled by $\left.\sqrt{k_{B} T / R_{\mathrm{ACN}}^{3}}\right)$ around $\mathrm{Li}^{+}$and $\mathrm{C}_{4}$ ions as a function of distance $r$ (scaled by water diameter $R_{\mathrm{ACN}}$ ) from the centre of the ion at $0 \cdot 10$ (solid line), $0 \cdot 50$ (short dashed line) and 0.70 (dotted dashed line) mole fractions of DMSO in DMSO-ACN mixtures. For discussion, see text. tures. While the upper panels describe the partial polarization densities of water around $\mathrm{Li}^{+}$and $\mathrm{C}_{4}$, those of ethanol are shown in the lower panels. Note that at the lowest ethanol mole fraction $(0 \cdot 10)$, both the first and second solvation shells are clearly visible whereas the second solvation shell disappears with the increase in alcohol mole fraction in the mixture. As expected, the peak value of the water polarization density decreases with the increase in ethanol concentration and vice-versa. The results for DMSOacetonitrile mixtures are shown in figure 3 . As observed in the ethanol-water mixture (figure 2), the polarization peak also decreases here as one increases the DMSO (larger component) concentration. However, the second solvation shell is well formed for most of the cases in this mixture. This is in contrast to what has been observed in ethanol-water mixtures (figure 2). This is again due to the larger size of the solvent components constituting the binary mixtures.

We show in figure 4 the mole fraction dependence of partial polarization peaks for these two mixtures. The decrease or increase of partial solvent polarization peak is non-linear for ethanol-water mixtures,
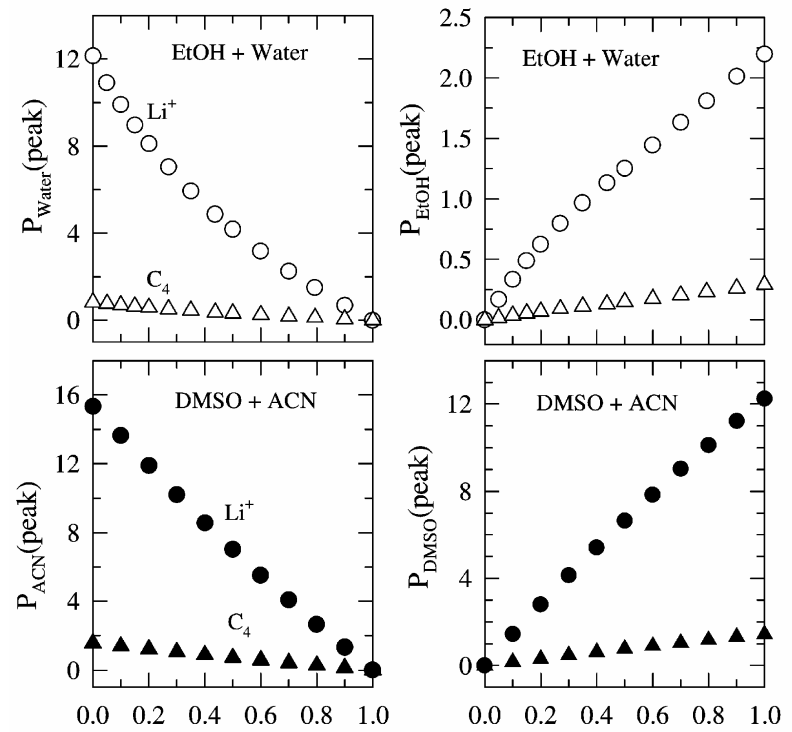

$\mathrm{x}_{2}(\mathrm{EtOH}$ or DMSO)

Figure 4. Upper panels: Comparison of peak values of partial polarization of water $P_{\text {water }}$ (peak) and ethanol $P_{\text {EtOH }}$ (peak) for $\mathrm{Li}^{+}$(open circles) and $\mathrm{C}_{4}$ (open triangles) ions as a function of mole fraction $x_{2}$ of ethanol in ethanol-water mixture. Lower panel: Comparison of peak values partial polarization of acetonitrile $P_{\mathrm{ACN}}$ (peak) and dimethylsulfoxide $P_{\text {DMSO }}$ (peak) for $\mathrm{Li}^{+}$(solid circles) and $\mathrm{C}_{4}$ (solid triangles) ions as a function of mole fraction $\mathrm{x}_{2}$ of DMSO in CAN-DMSO mixture. 
the extent of non-linearity being very weak for $\mathrm{C}_{4}$. As discussed earlier here and elsewhere ${ }^{76}$ the nonlinearity is a manifestation of preferential solvation and hence this explains the microscopic origin of the strong non-ideality observed for smaller ions in ethanol-water and other alcohol-water mixtures. In DMSO-acetonitrile mixture, however, the peaks of the partial solvent polarization densities decrease or increase almost linearly for both the ions showing a very weak preferential solvation. This is again due to the comparable sizes and dipole moments of DMSO and acetonitrile molecules.

\section{Conclusion}

Let us first summarize the main results of the paper. We have used the recently extended MSA formalism $^{76}$ to study the Born-free energy of solvation and non-ideality associated with it in associating (alcoholwater) and non-associating (DMSO-acetonitrile) solvent mixtures. The solvent dipoles are treated as dipolar hard spheres and ions as hard spheres with unit charge at centers. The electrolyte concentration has been infinitely dilute. The sizes of every species in the solution and the dipole moments of the different components have been systematically incorporated. The ethanol-water mixtures show greater non-ideality than DMSO-acetonitrile mixtures. The slope of the excess Born-free energy of solvation is found to be opposite in DMSO-acetonitrile mixture than that in ethanol-water solutions. The extremum positions of the excess Born-free energy of solvation versus mole fraction (of bigger component) curves have been found to be affected significantly by the solvent size ratio. Also, the larger ion exhibits smaller nonideality.

Since we treat these binary mixtures within the MSA framework, the present approach can neither distinguish between ions of different signs (cation or anion) nor can deal with any specific interactions and shape (say, spherical or ellipsoid) asymmetry that are present in real mixtures. Also, MSA is a linear theory which is known to fail for strongly polar systems. Therefore, the intra and inter-molecular correlations derived in this present work can be at best semiquantitative in nature. For an accurate description of these quantities one should use a better and nonlinear theories that are likely to be more numerically involved. The present approach, in contrast, is simple and analytically tractable yet captures the physics qualitatively correctly. This is definitely a positive aspect of the present work given the complexity of real solution mixtures.

\section{Acknowledgements}

We are deeply indebted to Profs Biman Bagchi and Kankan Bhattacharyya for their interest and constant encouragement. We sincerely thank Prof. Arup K Raychaudhuri for his constant support. We thank Ms $\mathrm{P}$ Ghoshal for experimentally measuring the mole fraction dependent densities of DMSO-acetonitrile mixtures. Financial assistance from the Council of Scientific and Industrial Research (CSIR), India and Department of Science and Technology (DST), India are gratefully acknowledged. HKK thanks SNBNCBS for a research fellowship.

\section{References}

1. Fleming G R and Wolynes P G 1990 Phys. Today 4336

2. Hynes J T 1985 The theory of chemical reactions (ed.) M Baer (CRC: Boca Raton) 4

3. Hynes J T 1985 Ann. Rev. Phys. Chem. 36573

4. Grote R F and Hynes J T 1980 J. Chem. Phys. 73 2715

5. Bagchi B and Biswas, R 1999 Adv. Chem. Phys. 109 207

6. Bagchi B and Gayathri N 1999 Adv. Chem. Phys. 1071

7. Biswas R and Bagchi B 1996 J. Chem. Phys. 105 7543

8. Nitzan A 1988 Adv. Chem. Phys. 70489

9. Bagchi B 1987 Int. Rev. Phys. Chem. 61

10. Bagchi B and Oxtoby D W 1983 J. Chem. Phys. 782735

11. Kim S K and Fleming G R 1988 J. Phys. Chem. 922168

12. Velsko S P, Waldeck D H and Fleming G R $1983 \mathrm{~J}$. Chem. Phys. 78249

13. Rothenberger G R, Negus D K and Hochstrasser R M 1983 J. Chem. Phys. 795360

14. Asano T, Furuta H and Sumi H 1994 J. Am. Chem. Soc. 165545

15. Sumi H and Asano T 1995 J. Chem. Phys. 1029565

16. Sumi H 1995 J. Mol. Liq. 6565

17. Berne B, Berkovec M and Straub J E 1988 J. Phys. Chem. 923711

18. Straub J E and Berne B 1986 J. Chem. Phys. 852999

19. Straub J E, Berkovec M and Berne B 1986 J. Chem. Phys. 841788

20. Pollak E J. Chem. Phys. 85865

21. Carmeli B and Nitzan A 1982 Phys. Rev. Lett. 49423

22. Carmeli B and Nitzan A 1983 Phys. Rev. Lett. 51233

23. Chandra A and Bagchi B 1991 Adv. Chem. Phys. 801

24. Bagchi B and Chandra A 1989 J. Chem. Phys. 90 7338

25. Bagchi B and Chandra A 1989 Chem. Phys. Lett. 155 533

26. Chandra A and Bagchi B 1989 J. Phys. Chem. 936996

27. Chandra A and Bagchi B 1989 J. Chem. Phys. 912954 
28. Chandra A and Bagchi B 1990 J. Phys. Chem. 94 3152

29. Biswas R and Bagchi B 1996 J. Phys. Chem. 1001238

30. Biswas R and Bagchi B 1996 J. Phys. Chem. 1001238

31. Biswas R and Bagchi B 1997 J. Chem. Phys. 1065587

32. Biswas R and Bagchi B 1997 J. Am. Chem. Soc. 119 5946

33. Biswas R, Nandi N and Bagchi B 1997 J. Phys. Chem. 1012968

34. Bagchi B and Biswas R 1998 Acc. Chem. Res. 31181

35. Chandra A 1995 Chem. Phys. Lett. 235 133; Chandra A 1998 Chem. Phys. 238285

36. Chowdhuri S and Chandra A 2003 J. Chem. Phys. 119 4360; Chowdhuri S and Chandra A 2005123 234501

37. Gupta R and Chandra A 2007 J. Chem. Phys. 127 024503

38. Day T J F and Patey G N 1999 J. Chem. Phys. 110 10937

39. Li H, Arzhantsev S and Maroncelli M 2007 J. Phys. Chem. B111 3208

40. Gardecki J A and Maroncelli M 1999 Chem. Phys. Lett. 301571

41. Broadwater T L and Kay R L 1970 J. Phys. Chem. 74 3802

42. Franks F and Ives D J G 1966 Q. Rev. Chem. Soc. 201

43. Zeidler M D 1973 In Water, a comprehensive treatise (ed.) F Franks (New York: Plenum) vol. 2, p. 529

44. Brai M and Kaatze U 1992 J. Phys. Chem. 908946

45. Kaatze U, Brai U, Scholle F D and Pottel R $1990 \mathrm{~J}$. Mol. Liq. 44197

46. Kaatze U, Pottel R and Schmidt P 1988 J. Phys. Chem. 92 3669; Kaatze U, Pottel R and Schmidt P 198993 5623; Kaatze U, Menzel K and Pottel R 1991 J. Phys. Chem. 95324

47. Wojkow D and Czarnecki M A 2005 J. Phys. Chem. A109 8218

48. Murthy S S N 1999 J. Phys. Chem. A103 7927

49. Iwasaki K and Fujiyama T 1979 J. Phys. Chem. 83 463; Iwasaki K and Fujiyama T 1977 J. Phys. Chem. 811908

50. Dixit S, Crain J, Poon W C K, Finney J L and Soper A K 2002 Nature (London) 416829

51. Soper A K and Finney J L 1993 Phys. Rev. Lett. 71 4346

52. Bowron D T, Soper A K and Finney J L $2001 J$. Chem. Phys. 1146203

53. Turner J and Soper A K 1994 J. Chem. Phys. 1016116

54. D'Angelo M, Onori G and Santucci A 1994 J. Chem. Phys. 1003107

55. Egashira K and Nishi N 1998 J. Phys. Chem. B102 4054

56. Yoshida K and Yamaguchi T 2001 Z. Naturforsch. A56 529

57. Nishikawa K, Kodera Y and Iijima T 1987 J. Phys. Chem. 913694

58. Koga Y 1984 Chem. Phys. Lett. 111176

59. Finney J L, Bowron D T and Soper A K $2000 \mathrm{~J}$. Phys. Condens. Matter 12 A123
60. Bowron D T, Finney J L and Soper A K $1998 \mathrm{~J}$. Phys. Chem. B102 3551

61. Bowron D T and Moreno S D 2005 J. Phys. Chem. B109 16210

62. Bowron D T and Moreno S D 2002 J. Chem. Phys 1173753

63. Euliss G W and Sorensen C M 1984 J. Chem. Phys. 804767

64. Sato T and Buchner R 2003 J. Chem. Phys. 119 10789

65. Tanaka H and Gubbins K 1992 J. Chem. Phys. 972626

66. Ferrario M, Haughney M, McDonald I R and Klein M L 1990 J. Chem. Phys. 935156

67. Palinkas G, Hawlicka E and Heinzinger K 1991 Chem. Phys. 15865

68. Wakisaka A, Komatsu S and Usui Y $2001 \mathrm{~J}$. Mol. Liq. 90175

69. Nakanishi K, Ikari K, Okazaki S and Touhara H 1984 J. Chem. Phys. 801656

70. Arnett E M and McKlevey D R $1966 \mathrm{~J}$. Am. Chem. Soc. $\mathbf{8 8} 5031$

71. Arnett E M and McKlevey D R $1965 \mathrm{~J}$. Am. Chem. Soc. 871393

72. Arnett E M, Bentrude W G, Burke J J and Duggleby P M 1965 J. Am. Chem. Soc. 871541

73. Srinivas G, Mukherjee A and Bagchi B $2001 \mathrm{~J}$. Chem. Phys. 1146220

74. Mukherjee A, Srinivas G and Bagchi B 2001 Phys. Rev. Lett. 865926

75. Gray C G and Gubbins K E 1984 Theory of molecular liquids (Oxford: Clarendon)

76. Kashyap H K and Biswas R 2007 J. Chem. Phys. 127 (submitted for publication)

77. Edward J T 1970 J. Chem. Edu. 47261

78. Chandra A and Bagchi B 1991 J. Chem. Phys. 94 8367

79. Morillo M, Denk C, Burgos F S and Sanchez A 2000 J. Chem. Phys. 1132360

80. Chan D Y C, Mitchel D J and Ninham B $1979 J$ Chem. Phys. 702946

81. Wertheim M S 1971 J. Chem. Phys. 554291

82. Isbister D and Bearman R 1974 J. Mol. Phys. 281297

83. Adelman S A and Deutch J M 1973 J. Chem. Phys. 593971

84. Blum L 1974 J. Chem. Phys. 612129

85. Cummings P T and Blum L 1986 J. Chem. Phys. 85 6658

86. Blum L and Wei D Q 1987 J. Chem. Phys. 87555

87. Wei D and Blum L 1987 J. Chem. Phys. 872999

88. Fort R J and Moore W R 1965 Trans. Faraday Soc. 612102

89. Densities of DMSO-acetonitrile binary mixtures at few mole fractions that were not available in ref. 88 have been determined experimentally by us using a compact density-cum-sound velocity analyzer (Model DSA5000) from Anton Paar, GmbH, Austria. Density data available in the ref. 88 have also been retaken in order to check the reproducibility.

90. Baxter R J 1970 J. Chem. Phys. 524559 